\title{
Robotic Management of Localized Adenocarcinoma Prostate with Large Vesical Calculus: A Report of Two Cases
}

\author{
${ }^{1}$ Girdhar Singh Bora, ${ }^{2}$ Prem Nath Dogra, ${ }^{3}$ Prabhjot Singh
}

\begin{abstract}
Introduction: Bladder outlet obstruction (BOO) accounts for more than $75 \%$ of cases of vesical calculi in patients aged above 50 years. There are special group of patients who have large vesical calculus with localized adenocarcinoma prostate requiring treatment for both bladder calculi and malignancy. We are sharing our technique of extraperitoneal robot-assisted radical prostatectomy (RRP) and removal of vesical calculus in two patients of localized adenocarcinoma prostate with large vesical calculus $(>4 \mathrm{~cm})$. Two patients with localized prostate cancer with large vesical stone underwent simultaneous cystolithotomy and extraperitoneal radical prostatectomy. Their perioperative period was uneventful. Large vesical stones with localized prostate cancer can be easily managed simultaneously by an experienced robotic surgeon.
\end{abstract}

Keywords: Bladder outlet obstruction, Robot-assisted radical prostatectomy, Vesical stone.

How to cite this article: Bora GS, Dogra PN, Singh P. Robotic Management of Localized Adenocarcinoma Prostate with Large Vesical Calculus: A Report of Two Cases. J Postgrad Med Edu Res 2016;50(1):36-38.

Source of support: Nil

Conflict of interest: None

\section{INTRODUCTION}

Bladder outlet obstruction (BOO) accounts for more than $75 \%$ of cases of vesical calculi in patients aged above 50 years. ${ }^{1}$ These vesical calculi should be treated along with the BOO either simultaneously or in a staged manner. Initially, vesical calculi were managed either by cystolitholapaxy for small stones or open cystolithotomy for large stones. With the advancements in endourology, vesical calculi are usually managed by percutaneous cystolithotomy or transurethral cystolithotripsy using holmium laser/pneumatic energy sources.

\footnotetext{
${ }^{1,3}$ Assistant Professor, ${ }^{2}$ Professor

${ }^{1}$ Department of Urology, Postgraduate Institute of Medical Education and Research, Chandigarh, India

${ }^{2,3}$ Department of Urology, All India Institute of Medical Sciences New Delhi, India

Corresponding Author: Prabhjot Singh, Assistant Professor Department of Urology, All India Institute of Medical Sciences, New Delhi, India, Phone: 09350773275, e-mail: drprbhjotsingh@gmail.com
}

Latest approaches like combined percutaneous cystolithotomy and transurethral resection of prostate or laparoscopic cystolithotomy with combined direct visual lithotripsy have evolved for large bladder stones with benign prostatic hyperplasia $(\mathrm{BPH}) .{ }^{2,3}$ There are special group of patients who have large vesical calculus with localized adenocarcinoma prostate requiring treatment for both bladder calculi and malignancy. However, there is a paucity of data regarding management of these complex cases. In this report, we are sharing our technique of extraperitoneal robot-assisted radical prostatectomy (RRP) and removal of vesical calculus in two patients of localized adenocarcinoma prostate with large bladder calculus $(>4 \mathrm{~cm})$.

\section{CASE DESCRIPTION}

Case 1: A 68-year-old male presented with both voiding and storage symptoms. His prostate specific antigen (PSA) was $9.12 \mathrm{ng} / \mathrm{ml}$ with clinical stage of T2a. Prostate biopsy revealed adenocarcinoma with Gleason score of $7(3+4)$. His KUB X-ray showed a single large $(4.1 \mathrm{~cm})$ vesical calculus (Fig. 1A).

Case 2: A 65-year-old male presented with voiding symptoms predominantly with PSA of $7.4 \mathrm{ng} / \mathrm{ml}$. His clinical stage was T1c. Prostate biopsy was adenocarcinoma with Gleason score of $6(3+3)$. KUB X-ray showed vesical stone of $4.3 \mathrm{~cm}$.

In both the cases, magnetic resonance imaging (MRI) pelvis was performed for staging.

\section{TECHNIQUE DESCRIPTION}

Initially, cystoscopy was done in both the patients for the confirmation of number of stones, status of bladder mucosa and identification of both ureteric orifices after placing the patients in lithotomy position. Oral phenazopyridine was given to both the patients preoperatively to confirm ureteric orifices intraoperatively. Preperitoneal space was created using spherical balloon (PDB, Autosuture) and 5 ports including one camera port $12 \mathrm{~mm}$ blunt tip trocar (BTT, Autosuture), 2 robotic $8 \mathrm{~mm}$ ports, one $12 \mathrm{~mm}$ assistant port on right-side and one $5 \mathrm{~mm}$ left-side port were placed. Patient was placed in $20^{\circ}$ trendelenburg position and robot was docked. Radical prostatectomy was performed first, followed by retrieval of stones. Initially, the endopelvic fascia opened bilaterally followed 
by ligation and division of deep venous complex with 2-0-monocryl suture. Anterior bladder neck at prostatovesical junction was incised followed by posterior bladder neck incision. Bilateral vas and seminal vesicles were dissected. Posterior dissection was completed by incising denonvilliers fascia and identifying prerectal fat. Incising urethra at apex completed radical prostatectomy and vesical stones were retrieved subsequently. In both the cases, stone remained at base of the bladder and did not migrate to dome area because of $20^{\circ}$ Trendelenburg position. Robotic ProGrasp forceps on right-side and Robotic Cadiere forceps on left-side were used for better grip of stones (Fig. 1B). Assistant helped in retraction of the anterior wall of bladder neck with the help of suction tip. Both robotic instruments were used to push the stone towards the bladder neck by applying pressure on the lateral walls. In the first case, anterior cystotomy was done for easy retrieval of stone. We reconstructed the cystotomy using 3-0 vicryl in racquet handle fashion. In second case, there was wide bladder neck during division of prostatovesical junction required bladder neck reconstruction. In both the patients, stone was placed
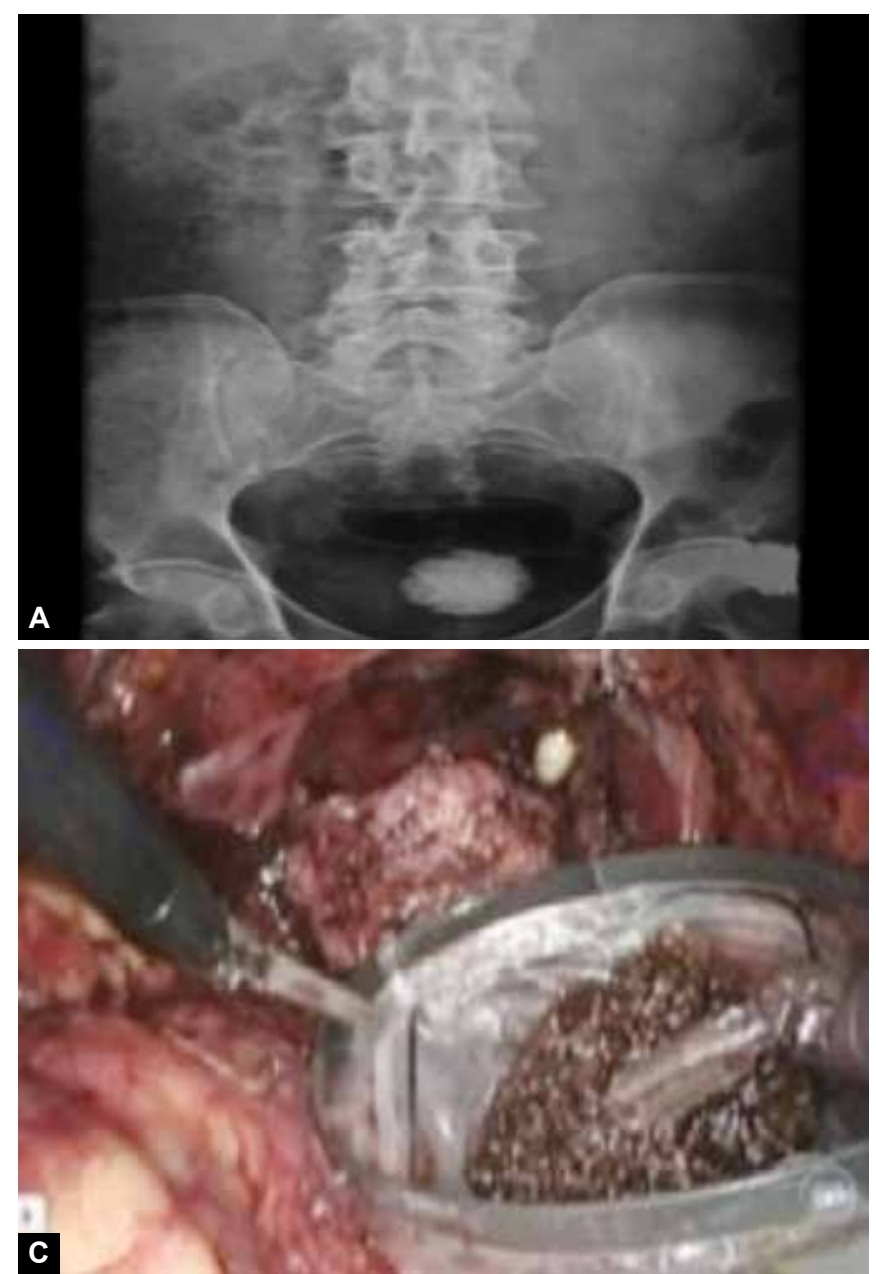

in same endocatch bag as for radical prostatectomy specimen (Fig. 1C). Both were retrieved through $12 \mathrm{~mm}$ assistant port incision (Figs 1D and E).

\section{RESULTS}

Mean surgeon console time was 150 minutes. Patients were allowed orally in the evening. Drain was removed on postoperative day 1 . Both the patients were discharged on third postoperative day. Final histopathology of radical prostatectomy specimen showed adenocarcinoma with Gleason score $7(4+3)$ in 1st patient and Gleason score $6(3+3)$ in 2 nd patient. All margins and seminal vesicles were free of tumor in both the patients. Mean follow-up of both the patients is 26 weeks and both the patients are continent and their PSA is undetectable.

\section{DISCUSSION}

Treatment options for large bladder calculi in patients due to benign prostatic hyperplasia are transurethral lithotripsy, percutaneous cystolithotripsy and open surgery. These procedures are associated with certain difficulties, like poor visual field due to bleeding and stone dust,
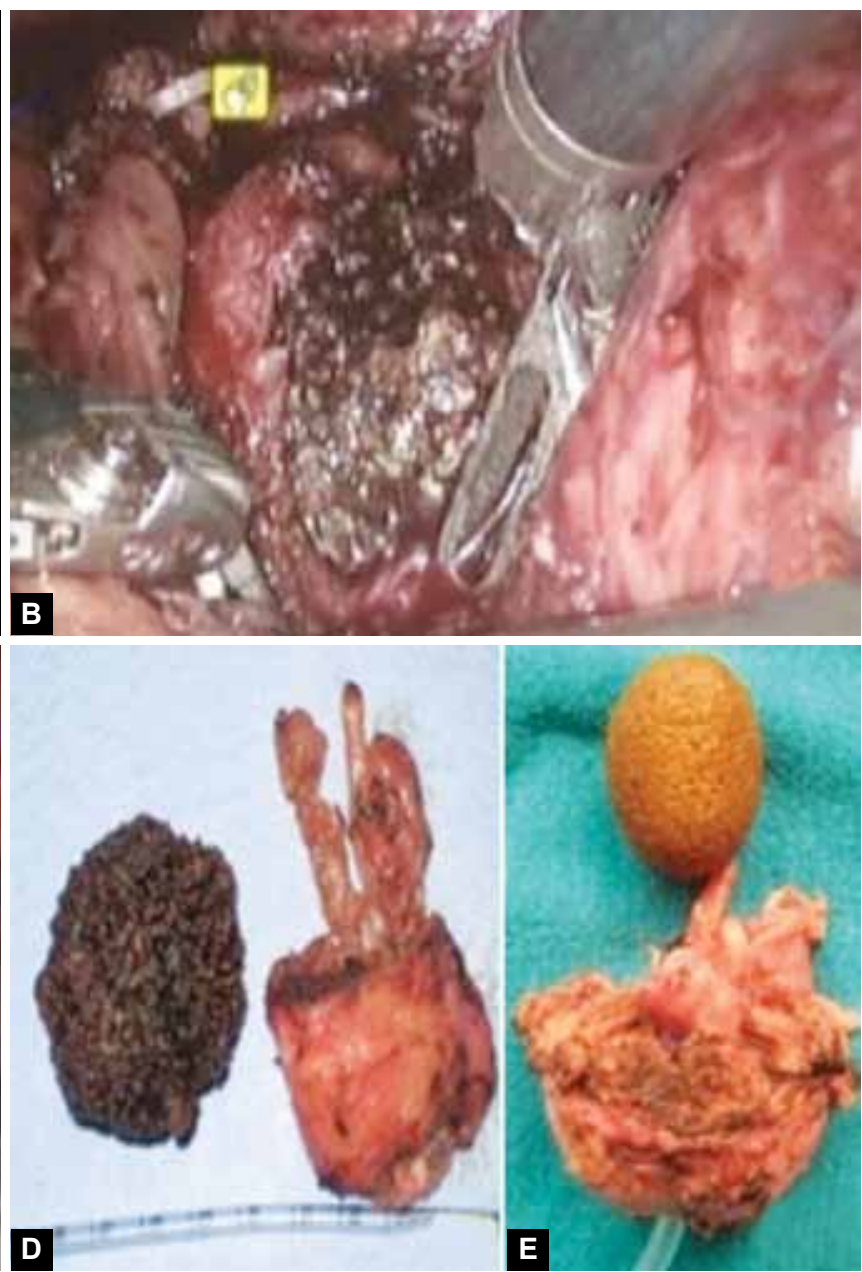

Figs $1 \mathrm{~A}$ to E: (A) KUB X-ray of patient 1 showing large vesical stone, (B) vesical stone with ProGrasp forceps on right-side and Cadiere forceps on left-side, $(C)$ vesical stone in endocatch bag, and ( $D$ and $E$ ) vesical stone and radical prostatectomy specimen of patients 1 and 2 
problems with stone fixation and complications, such as bladder and urethral trauma, urinary tract infection (lithotripsy in infected stones). ${ }^{4}$ It should be followed by either transurethral resection of prostate or open prostatectomy in the same sitting. In literature, there is scarcity of data regarding the management of large vesical stone with localized prostate cancer.

Radical prostatectomy is an effective treatment option for men with prostate cancer and offers the best long-term cancer control in patients with localized disease., ${ }^{5,6}$ With adequate learning curve, now RRP gives outcomes similar to open surgery. ${ }^{78}$ Patients with carcinoma prostate and large stones can be managed in a staged manner. In the first stage, stone can be managed transurethrally or percutaneously and radical prostatectomy can be done in the second stage. But it requires two sittings of anesthesia and possible complications of transurethral surgery including bladder mucosal injury and urethral injury leading to stricture urethra and difficult anterior bladder dissection following percutaneous surgery. With better maneuverability, increased degree of freedom due to robotic arms and experience, simultaneous RRP with retrieval of stones can be easily performed. It avoids trauma to bladder and urethra and ensures complete stone clearance.

Tan GY et $\mathrm{al}^{9}$ reported similar approach for patient with prostate cancer and bladder stone. But their case was different from our patient because their patient had multiple stones. They did not require bladder neck reconstruction. Larger stones can easily be removed by incision at the bladder neck. Stones can be trapped in an entrapment bag so that they can be taken out through a small working incision. There is always an apprehension that stone clearance should be complete. For that, we did the cystoscopy before RRP to confirm the number of stones. Otherwise, intraoperative flexible cystoscopy can be done to confirm complete clearance. We used the extraperitoneal approach with $20^{\circ}$ Trendelenburg position, which helped us for stone retrieval because stones remained at bladder base, and didn't migrate to dome, which usually happens in transperitoneal approach requiring steep Trendelenburg position. We used ProGrasps and cadiere forceps; both have serrated blades, which helped in retracting the bladder wall as well as retrieval of stone. These patients usually have inflamed bladder neck area and trigonal area due to stone. There may be difficulty in identifying ureteric orifices intraoperatively in these patients making them prone to injury during cystotomy. Cystoscopy before surgery with oral phenazopyridine preoperatively helped us to identify both the orifices intraoperatively.

\section{CONCLUSION}

Large vesical stones with localized prostate cancer can be easily managed simultaneously by an experienced robotic surgeon.

\section{REFERENCES}

1. Douenias R, Rich M, Badlani G, Mazor D, Smith A. Predisposing factors in bladder calculi: review of 100 cases. Urology 1991;37(3):240-243.

2. Richter S, Ringel A, Sluzker D. Combined cystolithotomy and transurethral resection of prostate: best management of infravesical obstruction and massive or multiple bladder stones. Urology 2002;59(5):688-691.

3. Lee JG, Yoo KH, Kim TH, Min GE, Jeon SH. A hybrid treatment for large bladder stones: laparoscopic cystolithotomy with combined direct visual lithotripsy. Kor J Urol 2009;50(9): 925-928.

4. Aron M, Agarwal MS, Goel A. Comparison of percutaneous with transurethral cystolithotripsy in patients with large prostates and large vesical calculi undergoing simultaneous transurethral prostatectomy. BJU Int 2003;91(3):293-295.

5. Roehl KA, Han M, Ramos CG, Antenor JAV, Catalona WJ. Cancer progression and survival rates following anatomical radical retropubic prostatectomy in 3,478 consecutive patients: long-term results. J Urol 2004;172(3):910-914.

6. Han M, Partin AW, Pound CR, Epstein JI, Walsh PC. Longterm biochemical disease-free and cancer-specific survival following anatomic radical retropubic prostatectomy: the 15-year Johns Hopkins experience. Urol Clin North Am 2001;28(3):555-565.

7. Ahlering TE, Skarecky D, Lee D, Clayman RV. Successful transfer of open surgical skills to a laparoscopic environment using a robotic interface: initial experience with laparoscopic radical prostatectomy. J Urol 2003;170(5):1738-1741.

8. Patel VR, Tully AS, Holmes R, Lindsay J. Robotic radical prostatectomy in the community setting - the learning curve and beyond: initial 200 cases. J Urol 2005;174(1):269-272.

9. Tan GY, Sooriakumaran P, Peters DL, Srivastava A, Tewari A. Cystolithotomy during robotic radical prostatectomy: singlestage procedure for concomitant bladder stones. Ind J Urol 2012 Jan;28(1):99-101. 